\title{
Arrested phase separation in a short-ranged attractive colloidal system: A numerical study
}

\author{
G. Foffi, $, 1,2$, C. De Michele, ${ }^{2,3}$ F. Sciortino, ${ }^{2,3}$ and P. Tartaglia ${ }^{2,4}$ \\ ${ }^{1}$ Institut Romand de Recherche Numérique en Physique des Matériaux IRRMA, PPH-Ecublens, CH-105 Lausanne, Suisse \\ ${ }^{2}$ Dipartimento di Fisica and INFM, Università di Roma La Sapienza, P.le A. Moro 2, 00185 Roma, Italy \\ 3 INFM - CRS Soft, Università di Roma La Sapienza, P.le A. Moro 2, 00185 Roma, Italy \\ 4 INFM - CRS SMC, Università di Roma La Sapienza, P.le A. Moro 2, 00185 Roma, Italy
}

\begin{abstract}
We numerically investigate the competition between phase separation and dynamical arrest in a colloidal system interacting via a short ranged attractive potential. Equilibrium fluid configurations are quenched at two different temperatures below the critical temperature and followed during their time evolution. At the lowest studied $T$, the phase separation process is interrupted by the formation of an attractive glass in the dense phase. At the higher $T$, no arrest is observed and the phase separation process proceeds endless in the simulated time window. The final structure of the glass retains memory of the interrupted phase separation process in the form of a frozen spinodal decomposition peak, whose location and amplitude is controlled by the average packing fraction. We also discuss the time evolution of the non ergodicity parameter, providing evidence of a progressively decreasing localization length on increasing the packing fraction. Finally, we confirm that the reported results are independent on the microscopic dynamics.
\end{abstract}

PACS numbers: 61.20.Lc, 64.70.Pf, 47.50.+d

\section{INTRODUCTION}

Advances in colloidal science make it possible to realize systems of interacting particles, with a tunable inter particle potential [1]. Interactions can be controlled in the range and in the strength, expanding considerably the possibilities found in atomic and molecular systems. The size of the colloidal particles allows experimentalist to study these systems with a wide range of optical techniques, such as Dynamic Light Scattering and Confocal Microscopy. Among the colloidal systems which have no atomic counterpart are the so-called short ranged attractive colloidal (SRAC) systems, in which the inter-particle potential has a range significantly smaller than the colloidal size. Experimentally these system can be realized by adding to a solution of colloidal particles a depletant agent, generally a polymer, When the radius of gyration is smaller than the colloidal size, the large particles experience an effective attraction whose range is related to the size of the polymer and the intensity to their concentration 2].

SRAC systems posses an extremely rich thermodynamic and dynamic behavior (for recent reviews see for example Refs. 3, 4, 5, 6). The short range attraction affects profoundly the structure of the phase diagram. When the range of the attraction is comparable to the particle size, the phase diagram presents a typical structure of a van der Waals fluid, i.e. a liquid-liquid critical point at low density and a solid-fluid phase transition at high density. However, when the range is much shorter than diameter of the colloidal particles, the liquid-liquid phase separation becomes metastable and is buried inside the fluid-

*Electronic address: giuseppe.foffi@epfl.ch solid coexistence curve. This phenomenon, predicted theoretically within perturbation theory $7,[8,[9]$, has been confirmed by simulation 10 and experiments 11, 12. It is interesting to note that critical fluctuations related to metastable critical point favor nucleation of the crystal phase 13.

Slow dynamic properties in SRAC systems also show features not observed in atomic systems. When short ranged attractions are present, the fluid-glass line (as well as its precursors, the iso-diffusivity lines 14, 15]) shows a reentrant behavior in the interaction strength-packing fraction plane. Two different competing mechanism for dynamic arrest are present, generating respectively a repulsive dominated and an attractive dominated glass. Dynamics become so complex that the usual stretched exponential decay characterizing the usual slowing down close to a a glass transition, cross over toward a logarithmic decay 16, 17, 18, 19, 20, 21]. These novel phenomena, first proposed on the basis of theoretical calculations based [16, 17] on the Mode Coupling Theory developed by Goetze and collaborators 22], have been subsequently confirmed by simulations 14, 15, 23, 24 and experiments 25, 26, 27, 28].

An additional phenomenon which is not usually encountered in atomic systems takes place at low packing fractions, where SRAC systems are known to form gel, i.e. arrested space spanning structure 29, 30, 31, 32, 33]. The nature of the gel transition in short-range attractive colloidal systems has received significant attention in recent years (for a recent review see for example Ref. 34]). Several routes to the gel state have been proposed and critically examined, with a special emphasis on the analogies and differences between glass and gel formation. It has been shown that, for some class of potentials, structural arrest at low packing fractions can be interpreted as a glass phenomenon of self-assembled clusters [35, 36, 37. 
For the case of SRAC, evidence is building that the gel state results from an arrested phase separation when the phase separation dynamics generate regions of local density sufficiently large to undergo an attractive glass transition 38, 39, 40, 41, 42, 43, 44].

In this manuscript we report results of Newtonian and Brownian molecular dynamics simulation of a binary mixture of particles interacting via a short range attractive square well. For this system, the location of the phase separation region and of the repulsive and attractive lines have been previously studied. The attractive glass line intersect the colloidal-rich colloidal-poor coexistence curve on the colloidal rich side at an intersection temperature $T_{x}$, a few per cent smaller than the critical temperature $T_{c}$. We quench initial configurations at different densities, equilibrated at a temperature where the system is homogeneous $\left(T>>T_{c}\right)$ to two temperatures lower then the critical one $\left(T<T_{c}\right)$ and study the coarsening dynamics. For the case of the higher final temperature, the coarsening dynamics never arrests and the systems slowly approached to equilibrium phase separated state characteristic of a conserved order parameter phase separation process. For lower temperture quench, we find that the coarsening dynamics arrests within the simulated time, leaving the system in an out-of-equilibrium arrested structure. The morphology of the resulting gel is controlled by the phase separation process at large distances (or small wave-vectors). At length scales comparable to the particle size, the structure resembles the one characteristic of the attractive glass.

\section{DETAILS OF THE NUMERICAL SIMULATIONS}

We investigate a system that has been extensively studied earlier, a binary square well (SW) mixture 15, 20]. The binary system is a $50 \%-50 \%$ mixture of $N=$ 2000 particles. The two species (labeled $A$ and $B$ ) are characterized by a diameter ratio $\sigma_{A} / \sigma_{B}=1.2$, which effectively suppress crystallization. Masses are chosen to be equal and unitary, i.e. $m_{a}=m_{b}=1$. The SW interaction is defined according to:

$$
V^{\alpha, \beta}(r)= \begin{cases}\infty & r<\sigma_{\alpha, \beta} \\ -u_{0} & \sigma_{\alpha, \beta}<r<\sigma_{\alpha, \beta}+\Delta_{\alpha, \beta} \\ 0 & r>\sigma_{\alpha, \beta}+\Delta_{\alpha, \beta}\end{cases}
$$

where $\sigma_{\alpha, \beta}=\left(\sigma_{\alpha}+\sigma_{\beta}\right) / 2, \alpha, \beta=A, B$ and $\Delta_{\alpha, \beta}$ is the range of the attraction. We fix $\sigma_{\alpha, \beta}$ and the wellwidth $\epsilon \equiv \frac{\Delta_{\alpha, \beta}}{\Delta_{\alpha, \beta}+\sigma_{\alpha, \beta}}=0.005$. The chosen $\epsilon$ value is arbitrary, but representative of all SW potential with interaction range smaller than a few percent as far as thermodynamic and dynamics equilibrium properties are concerned [43, 45]. For the chosen potential $T_{c} \approx 0.20$. We choose $k_{B}=1$ and set the depth of the potential $u_{0}=1$. Hence $T=1$ corresponds to a thermal energy $k_{B} T$ equal to the attractive well depth. The diameter of the small specie is chosen as unity of length, i.e.
$\sigma_{B}=1$. Density is expressed in term of packing fraction $\phi=\left(\rho_{A} \sigma_{A}^{3}+\rho_{B} \sigma_{B}^{3}\right) \cdot \pi / 6$, where $\rho_{\alpha}=N_{\alpha} / L^{3}, L$ being the box size and $N_{\alpha}$ the number of particles of specie $\alpha$. Time is measured in units of $\sigma_{B} \cdot\left(m / u_{0}\right)^{1 / 2}$. ND has been coded via a standard event driven algorithm, commonly used for particles interacting with step-wise potentials [46]. Between collisions, particles move along straight lines with constant velocities. When the distance between the particles becomes equal to the distance where the potential has a discontinuity, the velocities of the interacting particles instantaneously change. The algorithm calculates the shortest collision time in the system and propagate the trajectory from one collision to the next one. Calculations of the next collision time are optimized by dividing the system in small subsystems, so that collision times are computed only between particles in the neighboring subsystems. BD has been implemented via the position Langevin equation:

$$
\dot{\mathbf{r}}_{i}(t)=\frac{D_{0}}{k_{B} T} \mathbf{f}_{i}(t)+\stackrel{\circ}{\mathbf{r}}_{i}(t),
$$

coding the algorithm developed by Strating [47]. In Eq. [2 $\mathbf{r}_{i}(t)$ is the position of particle $i, \mathbf{f}_{i}(t)$ is the total force acting on the particle, $D_{0}$ is the short-time (bare) diffusion coefficient, $\stackrel{\circ}{\mathbf{r}}_{i}(t)$ a random thermal noise satisfying $<\stackrel{\circ}{\mathbf{r}}_{i}(t) \stackrel{\circ}{\mathbf{r}}_{i}(0)>=k_{B} T \delta(t)$. In Strating's algorithm, a random velocity (extracted from a Gaussian distribution of variance $\sqrt{k_{B} T / m}$ ) is assigned to each particle and the system is propagated for a finite time-step $\frac{2 m D_{0}}{k_{B} T}$, according to event-driven dynamics. We chose $D_{0}$ such that short time motion is diffusive over distances smaller than the well width.

Initial configurations were equilibrated at $T=1$ for densities ranging from $\phi=0.01$ to $\phi=0.50$. For each density, the system was quenched at two different final temperatures, $T_{f}=0.05<<T_{x}$ and $T_{f} \simeq 0.15 \simeq T_{x}$. The constant $T$ evolution was then followed in time. In the case of ND, the characteristic time of the thermostat as been chosen so that velocities reach thermal equilibrium before the system starts to rearrange its structural degrees of freedom.

\section{RESULTS AND DISCUSSION}

\section{A. Phase diagram}

The coexistence curve in the limit of vanishing well width, i.e. the celebrated Baxter limit 48], has been recently precisely calculated by Miller and Frenkel [49]. These data provides an accurate estimate of the coexistence curve of all short range interacting potential, via an appropriate law of corresponding-states [45]. Indeed, Noro and Frenkel noticed that, when the attraction range is much smaller than a few percent of the particles diameter, the value of the first virial coefficient at the critical temperature was essentially independent on the details of 
the potential, down to the Baxter limit. Following this prescription we reported in Fig. 1 the coexistence curve calculated by Miller and Frenkel in the $\tau$ - $\phi$ plane $(\tau$ is the Baxter stickiness parameter) after transforming it to the $T$ - $\phi$ plane according to

$$
1-\frac{1}{4 \tau}=\left[1-\left(e^{\beta u_{0}}-1\right)\left[(1-\epsilon)^{-3}-1\right]\right]
$$

i.e. imposing the equivalence of the virial coefficient of the Baxter and binary SW model. To independently support the mapping, we calculate explicitly the location of the spinodal line, by bracketing it with the highest $T$ at which no phase separation takes place and the lower $T$ at which we observe a growing spinodal decomposition peak. The calculated spinodal line, also reported in Fig. 1] is in very good agreement with Miller and Frenkel estimates.

Fig. [1 also shows isodiffusivity lines for the studied SW model, i.e. lines along which the normalized diffusion coefficient is constant 14]. These lines show the typical non monotonic behavior characteristic of SRAC systems, in which a speed up of the dynamics takes place at the temperature at which the competition between the two different arrest mechanisms is balanced. The isodiffusivity lines provide also a guide to the shape of the glass transition line, which has been numerically estimated, by extrapolation, as the zero isodiffusivity line 24]. As previously reported, the glass line meet the coexistence and spinodal line on the colloid-rich side, confirming that arrest in SRAC systems can take place at low packing fractions only as a result of an interrupted phase separation [42, 43].

Fig. 1also shows the location of the studied points in the phase diagram.

\section{B. Potential Energy - Number of Bonds}

One of the advantage of the SW models is represented by the fact that the potential energy per particle $U / N$ is directly related to the number of bonds $n_{b}$ per particle by $n_{b}=-2 U /\left(N u_{0}\right)$. Indeed two particle can be unambiguously considered bonded if their relative distance is within the attractive well distance. In the initial configurations, i.e. $T=1.0$, the potential energy varies between values characteristic of few particle bonded, for the lowest density, to less then one bond per particle, for $\phi=0.50$.

Fig. 22 reports the time evolution of $U / N$ for the ND case and $T_{f}=0.05$. Following the quench at $t=0$, the velocity degrees of freedom equilibrate and before $t \simeq 1.0$ the selected $T_{f}$ is reached. Structural degrees of freedom, however, relax on a much longer time scale, dragged by the tendency of the system to phase separate. The evolution of the energy shows three different processes. After a characteristic time, which is longer the smaller the initial packing fraction, aggregation sets in and the energy decreases significantly. This aggregation process slows down significantly once a number of bonds of the order of 6 per particle is reached. In this late stage of the simulations run, two different behaviors can be distinguished. At very low density, i.e . $\phi<0.05$, the energy continues to drop and does not seems to reach any stationary value in the simulation time window. For $\phi>0.05$, however, the time dependence of the energy abruptly stops and the system does not show sign of further evolution. This can be seen as first indication that an arrested structure has formed.

Fig. 20 reports data similar to the one presented in Fig. 2a but for BD. Despite the different microscopic dynamic, the time dependence of the energy shows similar trends and the value of the energy at which the system stops for different densities is indeed very similar to the ND case (see Fig. 3). Comparing in more details the two dynamics, one notice that the Brownian aggregation dynamics is smoother as compared to the Newtonian one, probably because in ND momentum conservation law require many body interactions for cluster aggregation. Two body interactions between two monomers can not produce a bounded dimer state. Once a small number of small clusters are present in the system, aggregation speeds up significantly.

Fig. 22 reports, for ND, the time dependence of the energy for the quench at $T_{f}=0.15$. Comparing Fig. 22 and Fig. 22, we notice that the major difference with the $T_{f}=0.05$ case is in the long time scale. In the present case, instead of remaining frozen, the energy continues to drift for all densities we considered (between $\phi=0.05$ and $\phi=0.50$ ) indicating that the separation process does not arrest during the simulated time.

The $\phi$-dependence of the final values of the energy is shown in Fig. 3a for the three cases discussed above. For the $T_{f}=0.05$ quench, on the overall range of densities this quantity varies between 3.1 and 2.8 and it presents a maximum close to the critical packing fraction. In equilibrium conditions, one would expect the energy to grow with the packing fraction but, in this out-of-equilibrium situation, this is note the case. To understand how the maximum arises, we show in Fig. 3b the distribution of bonds for three representative packing fraction, 0.10, $0.25,0.50$. While the high and the low density cases have roughly the same average number of bonds, at the critical packing fraction such an average is lower, as indeed expected by the value of the energy. This difference is originated by the presence of a large tail of particles with low number of bonds. In other words, at the critical packing fraction the aggregate, possess more surface particles than at lower and higher packing. This phenomenon may results from the fact that at the critical packing fraction critical fluctuations are stronger then at lower and higher packing.

It is interesting to note that, within numerical error, ND and $\mathrm{BD}$ results for $T_{f}=0.05$ are in agreement. Indeed this is not a trivial issue, since one would expect microscopic dynamics to play an important role in an out of equilibrium situation. 
For $T_{f}=0.15$, the final $U$ values are smaller than the ones at $T_{f}=0.05$, providing further evidence that at low $T$ a dynamic arrest transition has taken place, blocking the approach to an equilibrium phase separated state. Indeed, if equilibrium would have been reached, $U(T=0.05)$ should have been lower than $U(T=0.15)$.

\section{Structure of the aggregates in real space}

An insight of the evolution of the structure during the phase separation process can be gained by examining Fig. [4 where snapshots of the $\phi=0.10$ system are presented for different times, for $T_{f}=0.05$ (both for ND and BD) as well as for $T_{f}=0.15$. Before structural rearrangements start to take place (top row), the particles are homogeneously distributed within the simulation box. In the second set of snapshot, the phase separation process starts to create regions richer and lower in colloidal particle concentration (second row), which progressively coarsen (third row). In the final configurations (forth row), both the ND and $\mathrm{BD}$ results present a very similar ramified structure for the $T_{f}=0.05$ quench whilst, as expected, the structure for $T_{f}=0.15$ is more compact. Comparing these intermediate states with the last recorded one, it appears that for $T_{f}=0.05$ the system dynamics has arrested. For $T_{f}=0.15$, however, even if the system forms a percolating space-spanning structure, its evolution is not arrested. Indeed the system is still able to rearrange itself, suggesting the presence of a significant mobility.

It is important to stress that the arrested structures obtained by $\mathrm{ND}$ and $\mathrm{BD}$ are indeed very similar, which provides a support to the independence of the non-equilibrium structure to the microscopic dynamics. Thus, if one is interested, as in the present work, to characterize the arrested structure, it is possible to use equivalently BD or ND since the results is insensitive to the evolution scheme chosen. Obviously, since BD evolves toward the final structure roughly two order of magnitude slower then ND, the use of ND offers a net gain in terms of computational time. If one, however, is interested in the kinetic of the process, i.e. how the gel structure forms, issues concerning the microscopic dynamics should be properly addressed.

Fig. 5 and Fig. 5 show snapshots of the final configurations for packing fractions between 0.01 and 0.10 for the two quenches. At low temperature, i.e. $T_{f}=0.05$, and low packing fraction, i.e. $\phi=0.01$, the system is made up of large elongated cluster that does not span all the simulation box, i.e. does not percolate. Percolation is not observed at any stage during the separation process. Some monomers are still present, but their number progressively decreases with time. In real system the large aggregates will be eventually subject to precipitation due to the gravitational field. At $\phi=0.05$, the final structure percolates. All particles belong to the spanning cluster, which is extremely open and with tiny connections. The situation is even more evident at $\phi=0.10$. The network that span the box has a more defined structure and distribution of particle appears to be more uniform. This structure clearly resemble the idea of a gel, highly inhomogeneous percolating arrested structure. It is perhaps interesting to stress that the percolating structure is formed during the separation process.

For $T_{f}=0.15$, at low packing fraction, i.e. $\phi=0.01$, the final structure is similar to the previous case, the system has formed a single non percolating cluster. Its structure, however, is more compact than in the previous case resembling more the shape of spherical droplet. At $\phi=0.05$, the situation is different. A single cluster is present but it does not form anymore a rigid percolating structure but rather an elongated cluster. It is only at $\phi \geq 0.10$, that the systems percolates again. The general trend however, is that the structures are significantly more compact than for $T_{f}=0.05$, in agreement with the larger number of bonds (lower energy) observed at $T_{f}=0.15$ as compared to the $T_{f}=0.05$ case.

\section{Static structure factors}

To properly quantify the structure of the system we study the static structure factor as a function of the time elapsed after the quench. For a binary mixture the partial static structure factors, $S_{\alpha \beta}(q, t)$ can be defined as

$$
S_{\alpha \beta}(q, t)=\left\langle\varrho_{\alpha}^{*}(\mathbf{q}, t) \varrho_{\beta}(\mathbf{q}, t)\right\rangle
$$

where the partial density variables are defined as $\varrho_{\alpha}(\vec{q})=$ $\sum_{k=1}^{N_{\alpha}} \exp \left[i \mathbf{q} \cdot \mathbf{r}(t)_{k}^{(\alpha)}\right] / \sqrt{N}$. At equilibrium, time translation invariance implies $S_{\alpha \beta}(q, t)=S_{\alpha \beta}(q, 0)$, i.e. the static structure factor is a time independent function. In our case, however, we are in an out of equilibrium condition and the $t$ dependence must be maintained. In particular we shall focus on the total static structure factor defined as:

$$
S(q, t)=\sum_{\alpha, \beta}^{1,2} S_{\alpha \beta}(q, t)
$$

Numerically this quantity is calculated for a given configuration at a time $t$ by calculating the density variable $\varrho_{\alpha}(\vec{q})$ and averaging on different $q$-vectors with the same modulus $q$ but different orientation. Such spherical average was performed over up to 300 distinct $q$-vectors. Results for the ND $T_{f}=0.05$ case are shown in Fig. [6 for three different densities. $(\phi=0.10$ in Fig. 6a $)$, critical $(\phi=0.25$ in Fig. 6b) and high ( $\phi=0.50$ in Fig. [6) $)$.

At $\phi=0.10$, the structure factor starts from the typical shape of a low density hard sphere fluid, i.e. no structural peaks either at contact, i.e. $q \sim 6.2$ or at higher values. As the energy starts to drop, roughly at $t \sim 10^{2}$, the system becomes more structured. A first effect is represented by an increase at the nearest neighbor peak of the structure factor, a structural confirmation of bond formation. Simultaneously, a spinodal decomposition peak 
(at low $q$ ) starts to emerge. This indicates the development of a characteristic length roughly proportional to $2 \pi / q_{p}$, where $q_{p}$ is the location of the peak. This peak grows, coarsen and eventually stops.

To characterize the evolution of the peak we studied the evolution of the maximum and of the first moment $q_{1}$ of $S(q)$. This moment is defined as:

$$
q_{1} \equiv \frac{\int_{q}^{q_{c}} q S(q, t) d q}{\int_{0}^{q_{c}} S(q, t) d q}
$$

where the integral is performed with a proper cutoff $q_{c}$. This quantity scales as the peak location but can be calculated with better accuracy [42, 50]. We have chosen $q_{c}=4.0$, to weight the long wavelength fluctuations responsible for the phase separation. $S\left(q_{p}, t\right)$ and $q_{1}(t)$ are shown in the inset of Fig. 6a. The maximum of the peak start to grow at $t \sim 10^{2}$ and then at $t \sim 4 \times 10^{3}$ reaches a value around 20 and it remains frozen at this value. This is also confirmed by the behaviour of $q_{1}$ that after a transient time reaches a plateau. As discuss before 39, 40, 42 , these are phenomena that are typical of the formation of a dynamic arrested structure 31, 32].

For density close to the critical one, i.e. $\phi=0.25$, the behaviour is similar to the previous case, but less pronounced, as shown in Fig. 6b. $S(q, 0)$ presents a more pronounced contact peak, a consequence of the higher density. As the system evolves in time the contact peak grows as in the $\phi=0.10$ case. The dynamical evolution of the maximum and of the first moment $q_{1}$, shown in the inset of Fig. [ $\mathrm{b}$, is similar to the previous case but the final plateau values are much lower. Hence in this situation the arrested phase presents smaller aggregates and is more homogeneous then the case at lower density, quantifying the previous observations based on the real space pictures (see Sec. IIIC).

A different situation emerges when the system is at high density. The evolution of the structure factor for $\phi=0.50$ is presented in Fig. 6r. $S(q, 0)$ presents a very pronounced contact peak, higher then 2.5 due to the high particles packing. After the quench, when the system evolves, this first peak decreases and moves to slightly higher $q$, i.e. the opposite trend of the previous case. This is not surprising, it is well known that when the system at high density an temperature is cooled the effect for SRAC systems is the lowering of the first peak and the appearance of long tail oscillations. Indeed in $\mathrm{MCT}$ this trend is responsible for the reentrant glass line and for the formation of the attractive glass [18] and it has been observed in simulation on SW systems both at equilibrium [51] and in the aging regime 20]. It is interesting to note that, while the contact peak decreases with time, the second peak $(q \sim 12.5)$ increases, a clear indication of the emergence of these oscillations. Also for lower $q$, the situation is different from the two densities discussed above. A slightly increase of in the structure factor is present with time but now we can not talk of a real peak formation. The system freezes but its structure remains basically homogeneous and no large aggregates are formed, as confirmed by the evolution of the maximum and of $q_{1}$ reported in the inset of Fig. 6. These results suggest that at this packing fraction the system is sufficiently homogeneous that the MCT scenario is recovered. The frozen structure is similar to the one of an homogeneous liquid and the contact interactions are responsible for the structural arrest, enforcing the idea that SRAC gels are spatially inhomogeneous attractive glasses (in the MCT fashion), where the inhomogeneity is build by the phase separation process.

Analysis of the BD configurations confirms the above scenario, in agreement with the previous discussion based on the potential energy. Fig. 7f, Fig. 17b and Fig. (7) report $\mathrm{BD}$ results for $\phi=0.10, \phi=0.25$ and $\phi=0.50$ respectively. Results are very similar to that we previously discussed for ND. For $\phi=0.10$ and $\phi=0.25$ a low $q$ peak emerges and get arrested at a plateau. The difference with ND results is represented by the time it takes to get to the maximum value of the low $q$ peak. In the $\mathrm{BD}$, the transient is slower as was found in the energy evolution. Indeed this phenomenon is more evident for $\phi=0.10$, where in the explored simulation window, only the approach to the plateau can be studied. At $\phi=0.50$, no low- $q$ peak emerges in agreement with the ND results. The situation is different for the higher temperature quench case, i.e. $T_{f}=0.15$. Fig. $8 \mathrm{a}$, Fig. $8 \mathrm{~b}$ and Fig. 8 ; report $S(q, t)$ for $\phi=0.10,0.25,0.50$ respectively. As for the $T_{f}=0.05$ case, following the quench the system starts to develop inhomogeneities, expressed by the formation of a growing low $q$ peak in $S(q, t)$. In this case, however, no signs of structural arrest are observed. For example for $\phi=0.10$, Fig. 8 a, $S(q, t)$ develops a maximum at $t \sim 10^{3}$ that shows a slow but continuous increase. Similarly the position of the peak, represented by $q_{1}$, moves to lower $q$ and continues to drift for all the time of the simulation, indicating that the aggregates are growing in size. At later stage, $t \sim 4 \times 10^{4}$, the slow growth of the maximum presents a sharp increase giving evidence that a different regime has been reached. Similar behaviour is observed for $\phi=0.20$, Fig. 8 p, but in this case the increase in the maximum and position of the first peak is faster as expected since the quench is deeper in the phase separating region. Finally for $\phi=0.50$, Fig. 8k, there is an evident formation of a low $q$ peak that, differently from the $T_{f}=0.05$, slowly grows.

To conclude our analysis, we present $S(q)$ of final configurations at all the densities that have been investigated with ND. Fig. 9a shows the $T_{f}=0.05$ case. The first thing to notice is that the shape of the structure factors does not significantly depend on $\phi$ when $q \geq 5.0$, suggesting a similar local structure. At lower $q$, however, differences are evident. For the lowest density, $\phi=0.01$ and $\phi=0.05$, the system proceeds with the phase separation but for higher density this phenomenon is arrested. Moreover, increasing $\phi$ the degree of dis-homogeneity in the system decreases continuously up to $\phi=0.50$ where a low $q$ peak is not detected and the structure is very similar to that of homogeneous liquid. From this analysis it 
is evident that we can distinguish between two different $\phi$ regions. (i) At low packing fraction, i.e. $\phi<0.10$ the system does not present any arrest in the phase separating process. (ii) In the intermediate regime, i.e $0.10<\phi$, the system gets arrested in a structure that presents a lower degree of dis-homogeneity as the density is increased. The cross-over between these two regimes is characterized by the connectivity properties of the resulting structure. The onset of a percolating attractive glass structure appears to be the condition for global arrest. At small packing fractions, glass clusters can still freely diffuse and aggregate progressively. Formation of a gel built with a diffusion limited cluster mechanism in which the glassy clusters created in the phase separation process are the renormalized monomers is a possibility which can not be excluded, but it would requires the study of a much bigger system, which is out of our present capabilities. This issue remains open for further investigations.

In the $T_{f}=0.15$ case, the low $q$ regime grows indefinitely since at this value of the temperature the phase separation is taking over and the effect of the low temperature affects only the kinetics of this process.

\section{E. Structure of the aggregates in two dimensional slabs}

In this section we focus our attention on the properties of two dimensional slabs of the simulation box along one of the axis. This is something that, in general, is achieved in confocal microscopy experiments. Indeed, the typical size of a colloidal particle allows for this very powerful investigation in real space. Among the important phenomena that has been studied by this technique we can mention structural relaxation in hard sphere mixtures [52], nucleation phenomena [53], fluid-fluid interfacial properties 54 and indeed aggregation and gelation phenomenon [55, 56, 57.

We divide our simulation box in slabs along the $z$ axis. mentioning that in our simulation there is no external field acting on the system (such for example gravity) and consequently the choice of the height of the slabs is arbitrary. We take slabs of thickness $\Delta=4$, i.e. four times the diameter of the $B$ particles. We focus on the final configuration at $\phi=0.10$ and $T_{f}=0.05$, shown in Fig. [10 Fig. 11] shows snapshots from the top of three arbitrary slabs. The structure is extremely ramified and it is possible to notice long elongated clusters that form the basic unit of the arrested structure. As expected, the distributions of particles in the three slabs are very similar since the phase separating process was arrested before a big compact cluster was formed. The situation is different for the $T_{f}=0.15$ case. The three slabs present very different distributions, showing the presence of a big aggregate that results from the tendency of the system to form a single spherical droplet, since no arrest is taking place. On the contrary in a different slab, only few particles are present.
To better characterized this nearly two dimensional configuration, we calculate the two dimensional structure factor $S_{2 d}(q)$ defined as:

$$
S^{2 D}(q, z)=\sum_{\alpha, \beta}^{1,2}\left\langle\hat{\varrho}_{\alpha}^{*}(\hat{\mathbf{q}}), \hat{\varrho}_{\beta}(\hat{\mathbf{q}}, z)\right\rangle
$$

with,

$$
\hat{\varrho}_{\alpha}(\hat{\mathbf{q}})=\frac{1}{\sqrt{N(z)}} \sum_{k=1}^{N(z)} \exp \left[i\left(q_{x} x_{k}^{(\alpha)}+q_{y} y_{k}^{(\alpha)}\right)\right]
$$

where the sum is restricted to coordinates belonging to the particle that lies within $z+\Delta / 2$ and $z-\Delta / 2, N(z)$ is the number of such particles, $n_{\alpha}(z)$ the relative concentration of the two species in the slabs. The vector $\hat{\mathbf{q}}$, lies in the plane of the slabs and has the chosen modulus whereas the orientation is chosen randomly. For the total two dimensional structure factor a definition analogous to the one given by Eq. 5 holds. Fig [12] reports the results for $T_{f}=0.05$ for the three $z$-values considered together with the spherical averaged $S(q)$ considered before. In all cases in which the slab dimensions are representative of the sample, the three dimensional $S(q)$ coincides with the two-dimension one.

\section{F. Non ergodicity parameter}

When a system forms an arrested state only few of the possible configuration in phase space are actually explored, i.e. the system is non-ergodic. This is normally detected by the density correlation functions, $\phi_{q}^{\alpha \beta}\left(t, t_{w}\right)$ defined by:

$$
\phi_{q}^{\alpha \beta}\left(t, t_{w}\right)=\left\langle\varrho_{\alpha}^{*}(\mathbf{q}, t) \varrho_{\beta}\left(\mathbf{q}, t+t_{w}\right)\right\rangle
$$

where configuration at a time $t_{w}$ after the quench is correlated with a configuration at $t w+t$. In what follows we focus our attention on the total correlator defined, in a similar fashion to Eq. [5 by

$$
\phi_{q}\left(t, t_{w}\right)=\sum_{\alpha, \beta}^{1,2} \phi_{q}^{\alpha \beta}\left(t, t_{w}\right)
$$

When the system is at equilibrium, time translational invariance holds and the correlators are independent from the waiting time, i.e. $\phi_{q}^{\alpha \beta}\left(t, t_{w}\right)=\phi_{q}^{\alpha \beta}(t)$. When the system starts to lose ergodicity, correlators do not relax anymore to zero. This effect is usually measured in terms of the non ergodicity parameter $f_{q}$, defined as the long time limit of the correlator, i.e. $f_{q}=\phi_{q}(t \rightarrow \infty)$. This quantity represents the order parameter for the glass transition, since when it is zero the system is in an ergodic state, when it is finite the system is in a non-ergodic state. In equilibrium, within MCT formalism it is possible to directly calculate $f_{q}$ from the static structure factor and eventually test the theory with experimental or 
numerical results. We studied both the density-density correlation function and its long time value. We focus on the case $T_{f}=0.05$ and we chose to analyze the system closer to the critical packing fraction, i.e. $\phi=0.25$

In Fig. [13k, the evolution of the correlation function, expressed as $\phi_{q}\left(t-t_{w}\right)$, is presented for waiting times longer then the time needed to thermalized the velocity degree of freedom and for a representative $q$-vector, $q \sigma_{B}=20$. For short waiting time, the correlation functions relax to zero. For larger waiting time, a non ergodic contribution arises. Eventually, for large enough $t_{w}$, the correlators remains on a plateau that is very closed to unity. This behavior is different from the evolution of the aging dynamics for SRAC systems at higher density [20]. In that case, after a low temperature quench, there was no evident sign of a plateau even at the largest waiting time considered. In other words, the aging dynamics of the attractive glass is different from the present one, a fact that enforce the idea that the two phenomena have different origins.

The evolution of $f_{q}\left(t_{w}\right)$ with waiting time as obtained from the long time limit of the correlators is shown in Fig. 13] for four representative waiting times. The wavevector dependence of $f_{q}\left(t_{w}\right)$ develops progressively, starting from small $q$. The width of the non-ergodicity parameter is related to localization length. As time goes on, more and more particles aggregate, resulting in a decrease of the average localization length. The fact that already at short time the system posses a finite low $q$ nonergodicity parameter is a clear indication of the presence of a spanning structure made of few particles. As more particles join the percolating cluster the system becomes more non ergodic on shorter and shorter length scale. This suggests that the localization length progressively decreases, following the same pattern recently observed in both chemical [58] and thermoreversible [59] gels.

We have attempted to compare the non ergodic behavior observed in our simulations with MCT predictions, using as input the $S(q)$ calculated from the simulations. Unfortunately, as well known, MCT overestimates dynamical arrest. In the case of HS, arrest is predicted to take place for $\phi>0.516$ if the Percus-Yevich $S(q)$ is used and for $\phi>0.525$ with Verlet-Weiss correction or, more precisely, for $\phi>0.546$ is the "exact" $S(q)$ calculated from simulations is chosen 60]. In both cases, these critical $\phi$ values are smaller than the experimentally and numerically detected value of $\phi=0.58$. Similarly, the MCT predictions for the attractive glass overestimate the ideal glass transition temperature by more than a factor of two. Comparing experimental or simulation data with MCT predictions for the ideal glass transition locus requires an appropriate mapping in the $\phi-T$ plane [24, 61]. MCT predictions which do not account for the mapping suggest that the ideal glass line preempts the spinodal line (i.e. it is located above the phase separation curve) 62] Only when the appropriate mapping is accounted for the attractive glass line correctly ends in the high colloid concentration side of the spinodal curve [42]. It is very un- fortunate that the overestimate of the glass critical line prevents the possibility of meaningful solving the MCT equations for the case of the phase separating system by using as input the "exact" numerical $S\left(q, t_{w}\right)$, since the role of the large $q$ is dominating already at short time. Our attempts to solve the MCT equations failed in reproducing the small width observed in $f_{q}$ at short times $\left(t=57\right.$ in Fig. 13b). The first non vanishing $f_{q}$ was characterized by a width already larger than 50 (in units of $\left.q \sigma_{B}\right)$.

We perform a further analysis considering the variation of the final $f_{q}$ with density. The result is shown in Fig. 14 for a packing fraction ranging from 0.05 to 0.50 . The width of the non ergodicity parameter decreases when the packing fraction is decreased. This is an indication of the fact that the average localization length is larger for the lower packing fraction, the one characterized by a more open structure. As we discussed above, at high density the system maintains a certain homogeneity and no open empty region are detected. An open structure presents large amplitude modes and, as a consequence, the mean localization length is expected to grow with decreasing packing fraction. This interpretation is confirmed by the mean square displacement (MSD). In out of equilibrium, this quantity is defined as $\left\langle\left|\mathbf{r}\left(t-t_{w}\right)-\mathbf{r}\left(t_{w}\right)\right|^{2}\right\rangle$, where $\mathbf{r}(t)$ is the position of the particle at time $t$ and the average $\langle\cdot\rangle$ is performed over all the particles. Here we focus on the total MSD, i.e. evaluated with no distinction between particles of the two species for different values of $t+w$. Results are presented in Fig. 14b. After a ballistic short time region, the MSD reaches a plateau, whose value is larger the smaller $\phi$ is. Since the long-time value of the MSD provides an estimate of the characteristic size of the cages confining the particles, data in Fig.. 14b confirm that, in the arrested state, particles are more and more localized on increasing $\phi$.

\section{CONCLUSIONS}

The aim of this manuscript is to provide numerical evidence that, in the case of SRAC systems, the formation of an arrested state at low packing fraction results from a phase separation process interrupted by an attractive glass transition. We have shown that indeed, only when the system is quenched below $T_{x}$, the temperature at which the attractive glass line crosses the coexistence line on the high-polymer concentration side, the coarsening dynamics get arrested due to the mobility reduction associated to the glass transition. We also observed that the arrest takes place only when the structure is percolating, which at the studied $T_{f}$, requires $\phi \gtrsim 0.05$. For smaller $\phi$, diffusion of the droplets provides a slow coarsening mechanism which is missing in the percolating case. For $T>T_{x}$, we observed a continuous progressive coarsening process. The percolating ramified structures produced during 
the early stages of the phase separation process become thicker and thicker and the percolating cluster eventually collapses in the attempt of minimizing the surface area. These slow rearrangement processes are possible due to the residual mobility of the high $\phi$ phase.

The final structure of the aggregates as a function of $\phi$ is characterized by a peak at a finite wave-vector $q_{p}$, which constitute the frozen memory of the interrupted phase separation process. The location of the peak and its amplitude depend on $\phi$. The smaller the $\phi$, the lower is $q_{p}$ and the larger is $S\left(q_{p}\right)$. A complex interaction between the strength of the phase separation and the mobility in the dense phase define the final structure of the system. For example, for $\phi=0.50$, the system is so dense that it freezes before it can get significantly inhomogeneous and no considerable peak is detected in the static structure factor. It is also interesting to note that in this case, the evolution of $S(q)$ is not very different from the case of a quench in the attractive glass phase region [20], i.e. a decrease in the contact peak with waiting time. In the low density case the trend is the opposite, since more particles experience contact after the quench. It is also worth recalling that the connectivity of the frozen structure at particle level is also a function of $\phi$. We find that the number of bonds is minimized roughly in correspondence of the critical packing fraction. Perhaps this is related to the strength of the critical fluctuation, which increase the surface of the aggregate lowering, as a consequence the number of bonds.

Finally we studied the evolution of the non ergodicity parameter evaluated from the density-density correlation functions. We found that, differently from the aging dynamics in glasses, the non ergodicity parameter progressively increases with $t_{w}$. The first components to become significantly non ergodic are the large wavelength density fluctuations. The width of $f_{q}$ increases progressively during the coarsening dynamics. When dynamic arrest is completed, the localization length has become extremely narrow. Moreover it changes with packing fraction, showing less localized aggregates at lower density. The progressive increase of the $f_{q}$ width is reminiscent of the behavior recently observed in chemical [58] and thermoreversible 59] gel formation. A final remark is on the effect of the microscopic dynamics. In order to exclude any artifact introduced by Newtonian dynamics, we performed simulations, for the lowest $T$, also using Brownian dynamics. The two microscopic dynamics provide the same equilibrium description but different time scale. On approaching a structural glass transition, it has been shown that $\mathrm{ND}$ and $\mathrm{BD}$ generate the same long time behavior 63]. When the system is phase separating, as in our case, the microscopic dynamics could play a major role. We show, however, that the arrested structure obtained by the two different dynamical schemes are very similar. Since BD simulation can be a few order of magnitude slower than ND simulation, it is important to have the possibility to use the latter to describe aging processes in colloids.

\section{Acknowledgments}

G. F. acknowledges the support of the Swiss Science Foundation (Grant No. 99200021-105382/1). Support from MIUR-FIRB and Cofin and Training Network of the Marie-Curie Programmme of the EU (MRT-CT-2003504712) is acknowledged.
[1] W. B. Russel, D. A. Saville, and W. R. Schowalter, Colloidal Dispersions (Cambridge University Press, New York, 1989).

[2] C. N. Likos, Physics Reports 348, 267 (2001).

[3] V. J. Anderson and H. N. W. Lekkerkerker, Nature 416, 811 (2002).

[4] L. Cipelletti (2005), to be published in J. Phys.: Condens. Matter.

[5] D. Frenkel, Science 296, 65 (2002).

[6] F. Sciortino, Nature Materials 1, 145 (2002).

[7] A.P.Gast, W. Russell, and C. Hall, J. Colloid Interface Sci. 96, 1977 (1983).

[8] C. F. Tejero, A. Daanoun, H. N. W. Lekkerkerker, and M. Baus, Phys. Rev. Lett. 73, 752 (1994).

[9] M. Dijkstra, J. Brader, and R. Evans, J.Phys.:Condens. Matter 11, 10079 (1999).

[10] M. Hagen and D. Frenkel, J. Chem.Phys 101, 4093 (1994).

[11] H. N. W. Lekkerkerker, W. C. K. Poon, P. N. Pusey, A. Stroobants, and P. B. Warren, Europhys. Lett. 20, 559 (1992).
[12] S. Illett, A. Orrock, W. Poon, and P.N.Pusey, Phys. Rev E 51, 1344 (1995).

[13] S. Auer and D. Frenkel, Nature 409, 1020 (2001).

[14] G. Foffi, K. A. Dawson, S. V. Buldyrev, F. Sciortino, E. Zaccarelli, and P. Tartaglia, Phys. Rev. E 65, 050802(R) (2002).

[15] E. Zaccarelli, G. Foffi, K. A. Dawson, S. V. Buldyrev, F. Sciortino, and P. Tartaglia, Phys. Rev. E 66, 041402 (2002).

[16] L. Fabbian, W. Götze, F. Sciortino, P. Tartaglia, and F. Thiery, Phys. Rev. E 59, R1347 (1999).

[17] J. Bergenholtz and M. Fuchs, Phys. Rev. E 59, 5706 (1999).

[18] K. A. Dawson, G. Foffi, M. Fuchs, W. Götze, F. Sciortino, M. Sperl, P. Tartaglia, T. Voigtmann, and E. Zaccarelli, Phys. Rev. E 63, 011401 (2001).

[19] M. Sperl, Phys. Rev. E 68, 031405 (2003).

[20] G. Foffi, E. Zaccarelli, S. Buldyrev, F. Sciortino, and P. Tartaglia, J. Chem. Phys. 120, 8824 (2004).

[21] W. Götze and M. Sperl, J. Phys.: Condens. Matter 16, S4807 (2004). 
[22] W. Götze, in Liquids, Freezing and Glass Transition, edited by J. P. Hansen, D. Levesque, and J. ZinnJustin (North Holland, Amsterdam, 1991), vol. Session LI (1989) of Les Houches Summer Schools of Theoretical Physics, pp. 287-503.

[23] A. M. Puertas, M. Fuchs, and M. E. Cates, Phys. Rev. Lett. 88, 098301 (2002).

[24] F. Sciortino, P. Tartaglia, and E. Zaccarelli, Phys. Rev. Lett. 91, 268301 (2003).

[25] F. Mallamace, P. Gambadauro, N. Micali, P. Tartaglia, C. Liao, and S.-H. Chen, Phys. Rev. Lett. 84, 5431 (2000).

[26] K. N. Pham, A. M. Puertas, J. Bergenholtz, S. U. Egelhaaf, A. Moussaïd, P. N. Pusey, A. B. Schofield, M. E. Cates, M. Fuchs, and W. C. K. Poon, Science 296, 104 (2002).

[27] T. Eckert and E. Bartsch, Phys. Rev. Lett. 89, 125701 (2002).

[28] S.-H. Chen, W.-R. Chen, and F. Mallamace, Science 300, 619 (2003).

[29] W. C. K. Poon, Curr. Opin. Colloid Interf. Sci. 3, 593 (1998).

[30] N. A. M. Verhaeg, D. Asnaghi, and H. Lekkerkerker, Physica A 264, 64 (1999).

[31] P. N. Segrè, V. Prasad, A. B. Schofield, and D. A. Weitz, Phys. Rev. Lett. 86, 6042 (2001).

[32] S. A. Shah, Y.-L. Chen, S. Ramakrishnan, K. S. Schweizer, and C. F. Zukoski, J. Phys.: Condens. Matter 15, 4751 (2003).

[33] H. Sedgwick, S. U. Egelhaaf, and W. C. K. Poon, J. Phys.: Condens. Matter 16, S4913 (2004).

[34] V. Trappe and P. Sandkühler, Curr. Opin. Colloid Int. Sci. 8, 494 (2004).

[35] F. Sciortino, S. Mossa, E. Zaccarelli, and P. Tartaglia, Phys. Rev. Lett. 93, 055701 (2004).

[36] N. Sator, A. Fierro, E. D. Gado, and A. Coniglio (2004), cond-mat/0309007.

[37] K. Kroy, M. E. Cates, and W. C. K. Poon, Phys. Rev. Lett. 92, 148302 (2004).

[38] M. T. A. Bos and J. H. J. van Opheusden, Phys. Rev. E 53, 5044 (1996).

[39] K. G. Soga, J. R. Melrose, and R. C. Ball, J Chem. Phys. 108, 6026 (1998).

[40] J. Lodge and D. Heyes, Phys. Chem. Chem. Phys 1, 2119 (1999).

[41] R. J. M. d'Arjuzon, W. Frith, and J. R. Melrose, Phys. Rev. E 67, 061404 (2003).

[42] E. Zaccarelli, F. Sciortino, S. Buldyrev, and P. Tartaglia, in Unifyng Concepts in Granular Media and Glasses, edited by A. Coniglio, A. Fierro, H. Herrmann, and M. Nicodemi (Elsevier, Amsterdam, 2004).

[43] G. Foffi, C. De Michele, F.Sciortino, and P. Tartaglia, cond-mat/0410358.

[44] S. Manley, H. M. Wyss, K. Miyazaki, J. Conrad, V. Trappe, L. Kaufman, D. Reichman, and D.A.Weitz (2005), cond-mat/0309007.

[45] M. Noro and D. Frenkel, J.Chem.Phys. 113, 2941 (2000).

[46] D. C. Rapaport, The art of computer simulations (Cambridge Univ Press, London, 1997), 2nd ed.

[47] P. Strating, Phys. Rev. E 59, 2175 (1999).

[48] R. J. Baxter, J. Chem. Phys. 49, 2770 (1968).

[49] M. A. Miller and D. Frenkel, Phys. Rev. Lett. 90, 135702 (2003).

[50] S. C. Glotzer, M. F. Gyure, F. Sciortino, A. Coniglio, and H. E. Stanley, Phys. Rev. E 49, 247 (1994).

[51] E. Zaccarelli, G. Foffi, K. A. Dawson, S. V. Buldyrev, F. Sciortino, and P. Tartaglia, J. Phys.: Condens. Matter 16, S367 (2003).

[52] E. R. Weeks, J. C. Crocker, A. C. Levitt, A. Schofield, and D. A. Weitz, Science 287, 627 (2000).

[53] U.Gasser, E. Weeks, A. Schofield, P. Pussey, and D. Weitz, Science 292, 258 (2001).

[54] D. G. A. L. Aarts, M. Schmidt, and H. N. W. Lekkerkerker, Science 304, 847 (2004).

[55] M. L. Kilfoil, E. E. Pashkovski, J. A. Masters, and D. A. Weitz, Philosophical Transactions: Mathematical, Physical and Engineering Sciences 361, 753 (2003).

[56] H. Sedgwick, S. U. Egelhaaf, , and W. C. K. Poon, J. Phys: Cond. Mat. 16, S4913 (2004).

[57] A. Stradner, H. Sedgwick, F. Cardinaux, W. C. K. Poon, S. U. Egelhaaf, and P. Schurtenberger, Nature 432, 492 (2004).

[58] I. Saika-Voivod, E. Zaccarelli, S. Buldyrev, F. Sciortino, and P. Tartaglia, Phys. Rev. E 70, 041401 (2004).

[59] E. Zaccarelli, S. V. Buldyrev, E. L. Nave, A. J. Moreno, I. Saika-Voivod, F. Sciortino, and P. Tartaglia, condmat/0409361.

[60] G. Foffi, W. Götze, F. Sciortino, P. Tartaglia, and T. Voigtmann, Phys. Rev. E 69, 011505 (2004).

[61] M. Sperl, Phys. Rev. E 68, 031405 (2003).

[62] J. Bergenholtz, W. Poon, and M. Fuchs, Langmuir 19, 4493 (2003).

[63] T. Gleim, W. Kob, and K. Binder, Phys. Rev. Lett. 81, 4404 (1998). 


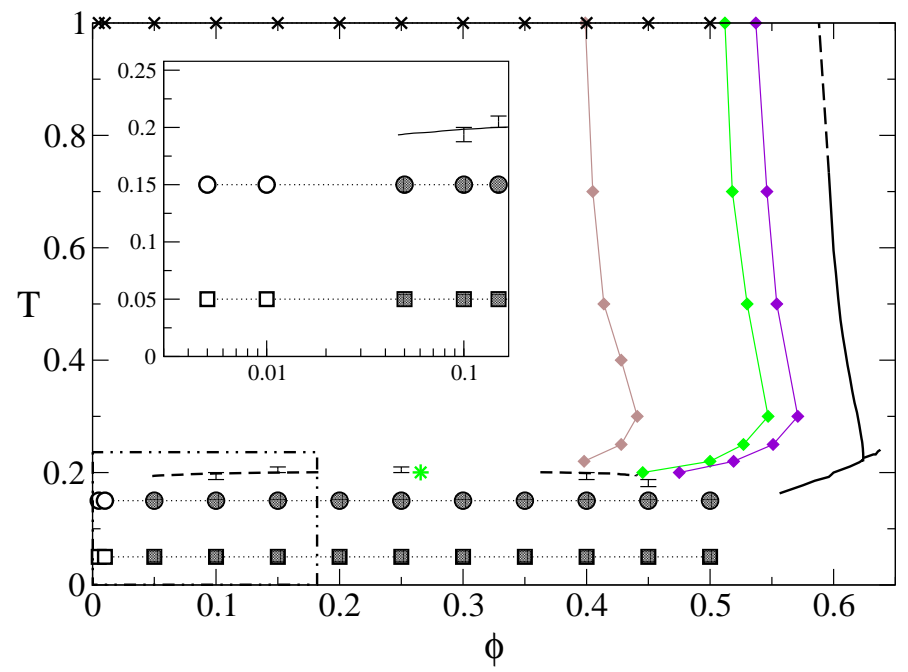

FIG. 1: Graphical representation of the studied state points in the temperature-packing fraction plane. Crosses represent the equilibrium starting configurations at $T=1$, which, at time zero, are quenched at $T_{f}=0.15$ (circles) and $T_{f}=0.05$ (squares) respectively. Symbols are shaded if, at the end of the simulation, the structure of the system is percolating. The error bars, calculated as explained in the text, provide an indication of the spinodal curve for the studied model. The present estimates are consistent with the phase coexistence calculations of Miller and Frenkel [49] for the Baxter model (dashed line), properly transformed using the virial mapping (Eq 3). The red-star indicates the location of the critical point, from Ref. [49]. To better frame the location of the studied state points, the Figure also shows three calculated isodiffusivity curves, i.e. the locus of points at which the diffusion coefficient $D / D_{0}=5 \times 10^{-2}, 1 \times 10^{-2}$ and $5 \times 10^{-3}$ with $\left.D_{o}=\sigma_{B} \sqrt{(} T / M\right)$. The bold line is the extrapolation to $D / D_{0} \rightarrow 0$ from Ref. [42], rescaled to the present well width case using Eq 3 The extrapolated $D / D_{o} \rightarrow 0$ line (see Ref. 24]) provides an estimate of the location of line of dynamical arrest for this system. 

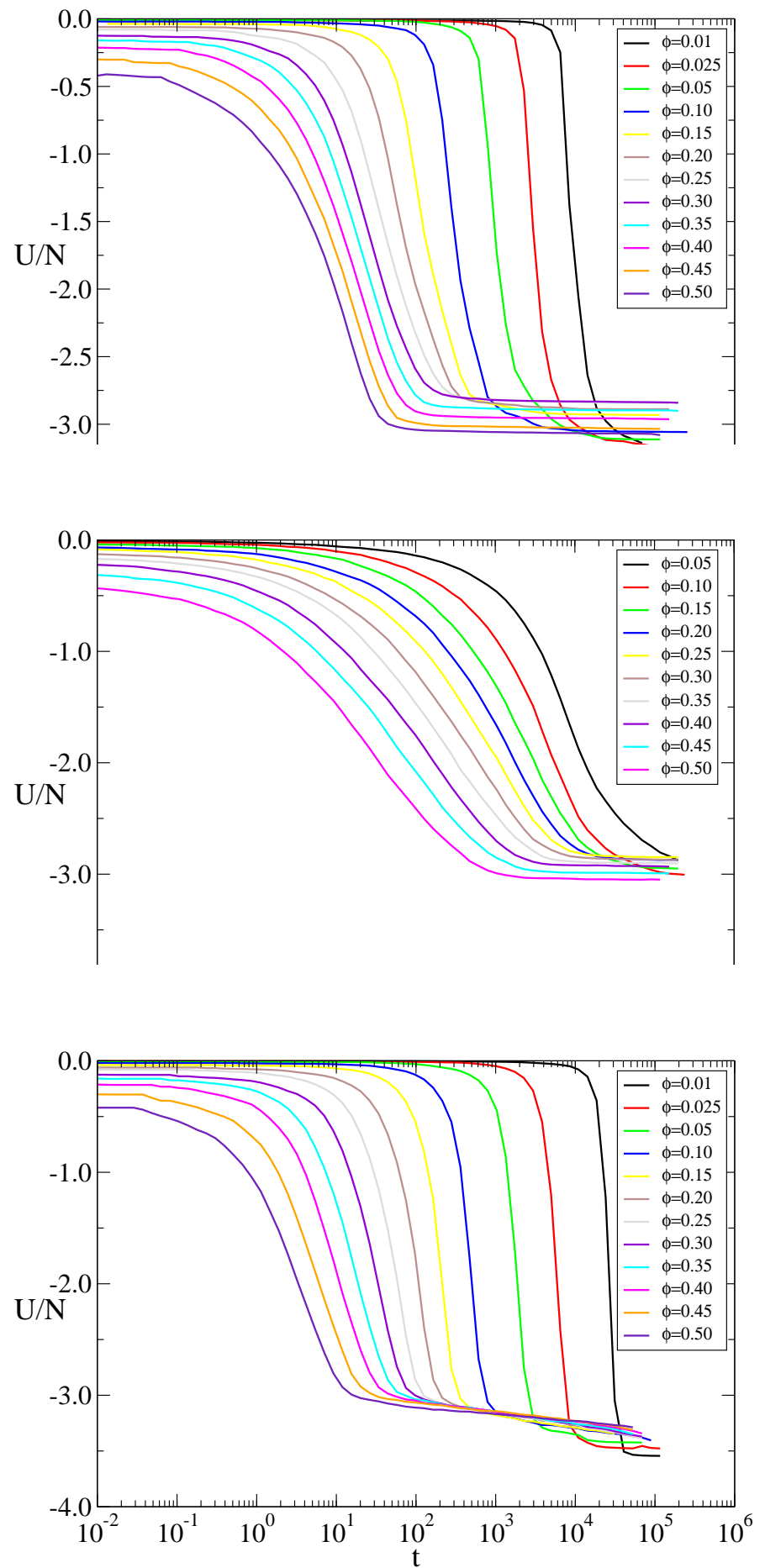

FIG. 2: (a) Time evolution of the potential energy per particle $U / N$ at $T_{f}=0.05$, following the quench from $T=1$, for different packing fractions. (b) Same as (a) but for Brownian dynamics. (c) Same as (a) but for $T_{f}=0.15$. 

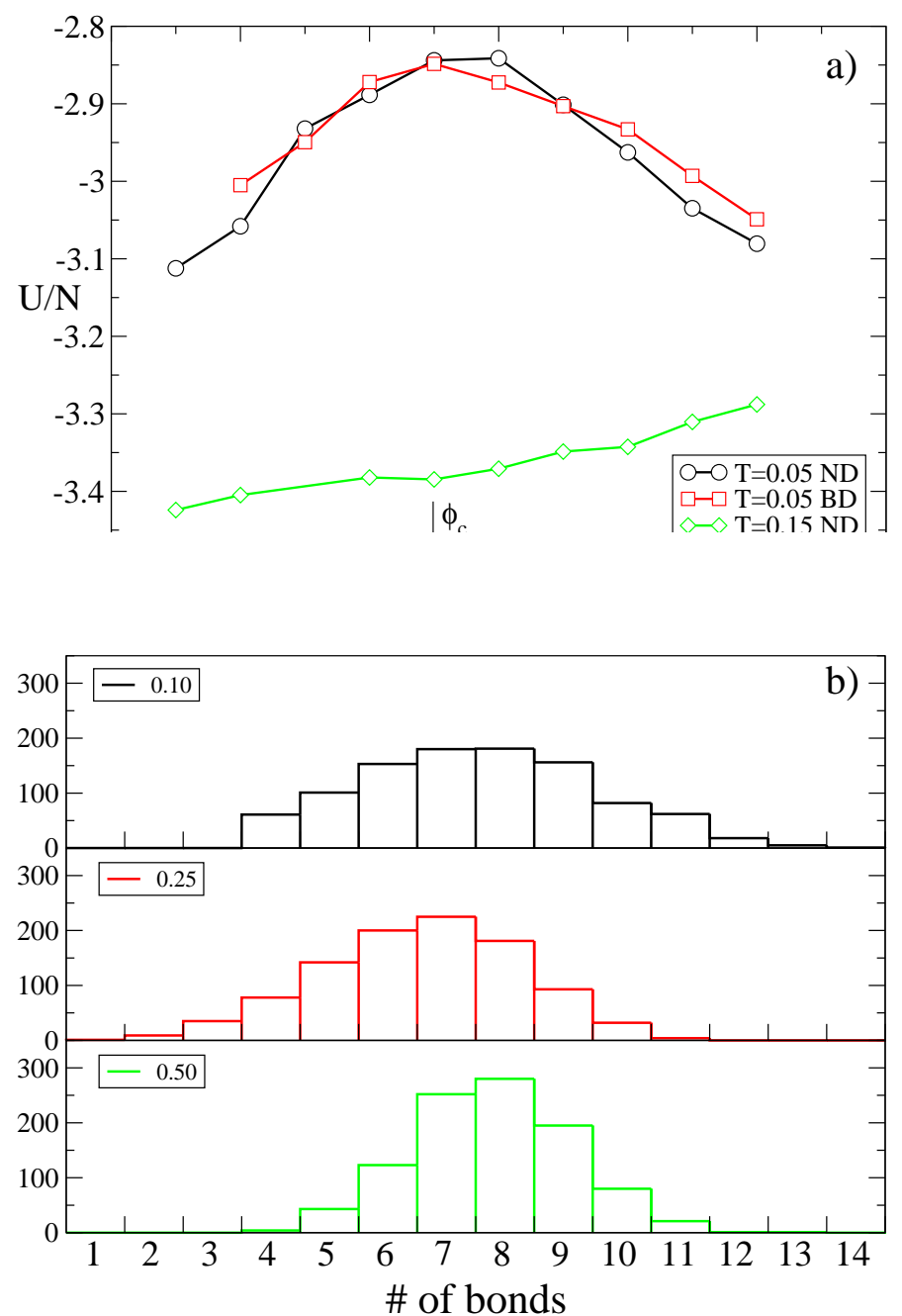

FIG. 3: (a) Potential energy per particle at the end of the simulation versus $\phi$ for $T_{f}=0.05$ (Newtonian and Brownian dynamics) and for $T_{f}=0.15$ (Newtonian). (b) Histograms of the distribution of bonds per particle for three packing fractions: $\phi=0.10, \phi=0.25, \phi=0.50$.

FIG. 4: Snapshots of the configurations at different times from the quench at $T_{f}=0.05$ and $\phi=0.10$ : (a) Newtonian Dynamics and (b) Brownian Dynamics. (c) same as (a) for $T_{f}=0.15$ and $\phi=0.10$

$$
\left(\mathbf{T}_{f}=0.05\right) \quad\left(T_{f}=0.15\right)
$$

FIG. 5: Snapshots of the final configurations for $T_{f}=0.05$ (top left) and $T_{f}=0.15$ (top right) for $0.01<\phi<0.5$. The unity of length $\left(\sigma_{b}\right)$ is graphically kept constant for the first and the last three rows. Hence, an increase in density implies a smaller simulation box. 

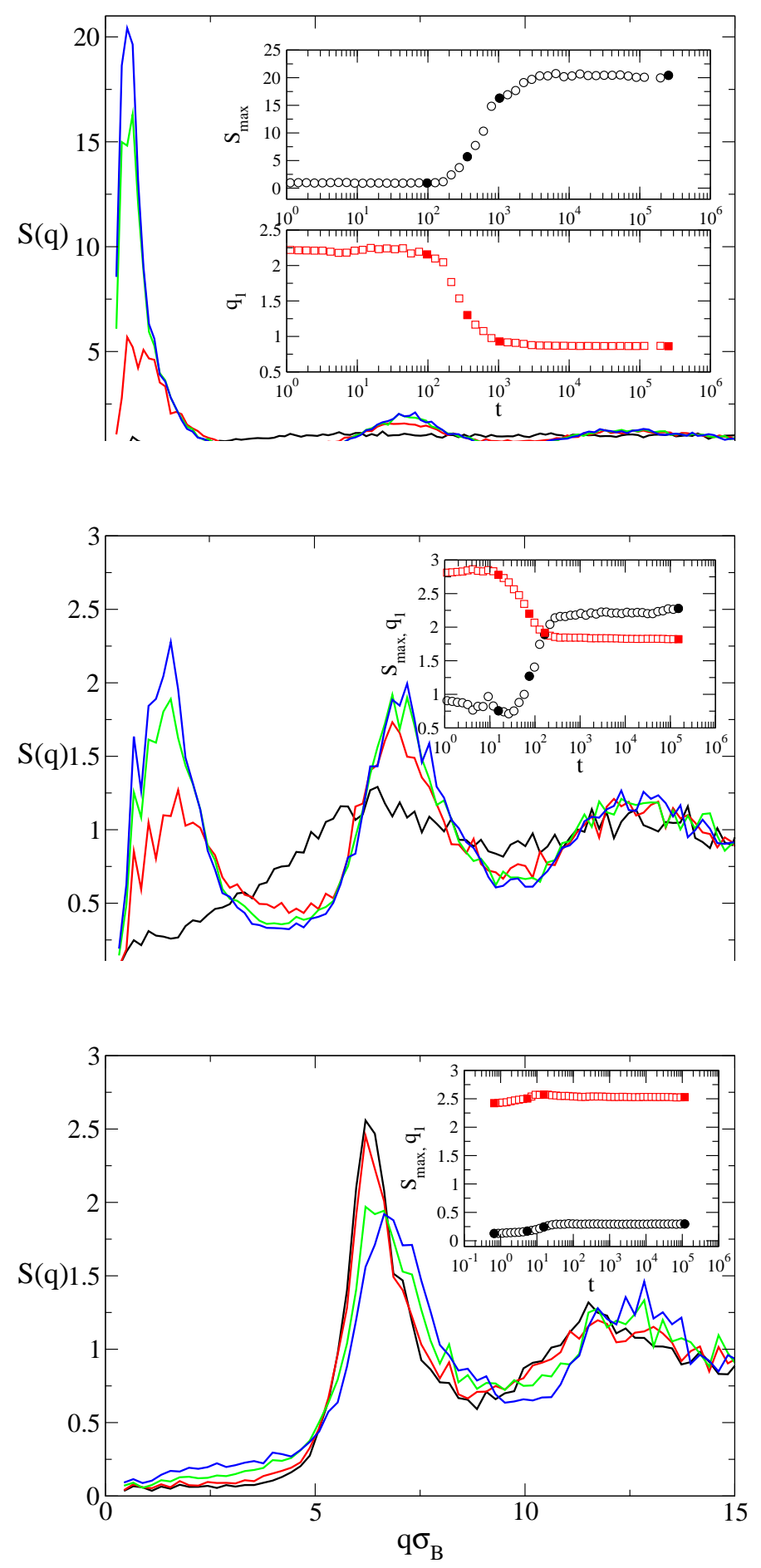

FIG. 6: Time evolution of the static structure factor $S(q)$ for $T_{f}=0.05$ at three packing fractions: (a) $\phi=0.10$, (b) $\phi=0.25$ (the critical packing fraction), (c) $\phi=0.50$. In the inset evolution of the height of the first maximum and its position expressed by the quantity $q_{1}$ is shown(see text for details). The full symbols in the inset represent the times at which the structure factors are shown in the main figure. 

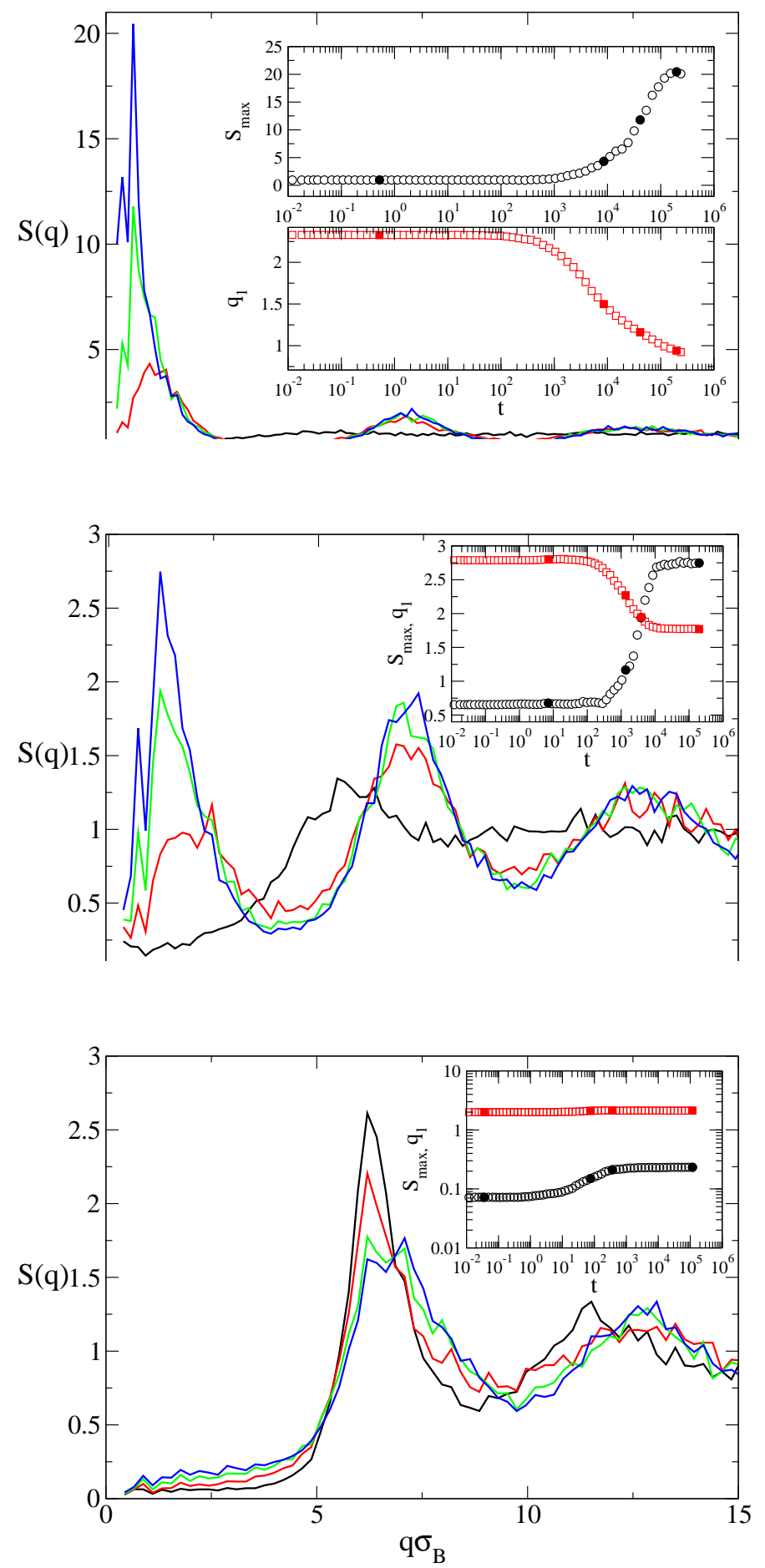

FIG. 7: Same as Fig [6] for Brownian Dynamics. 

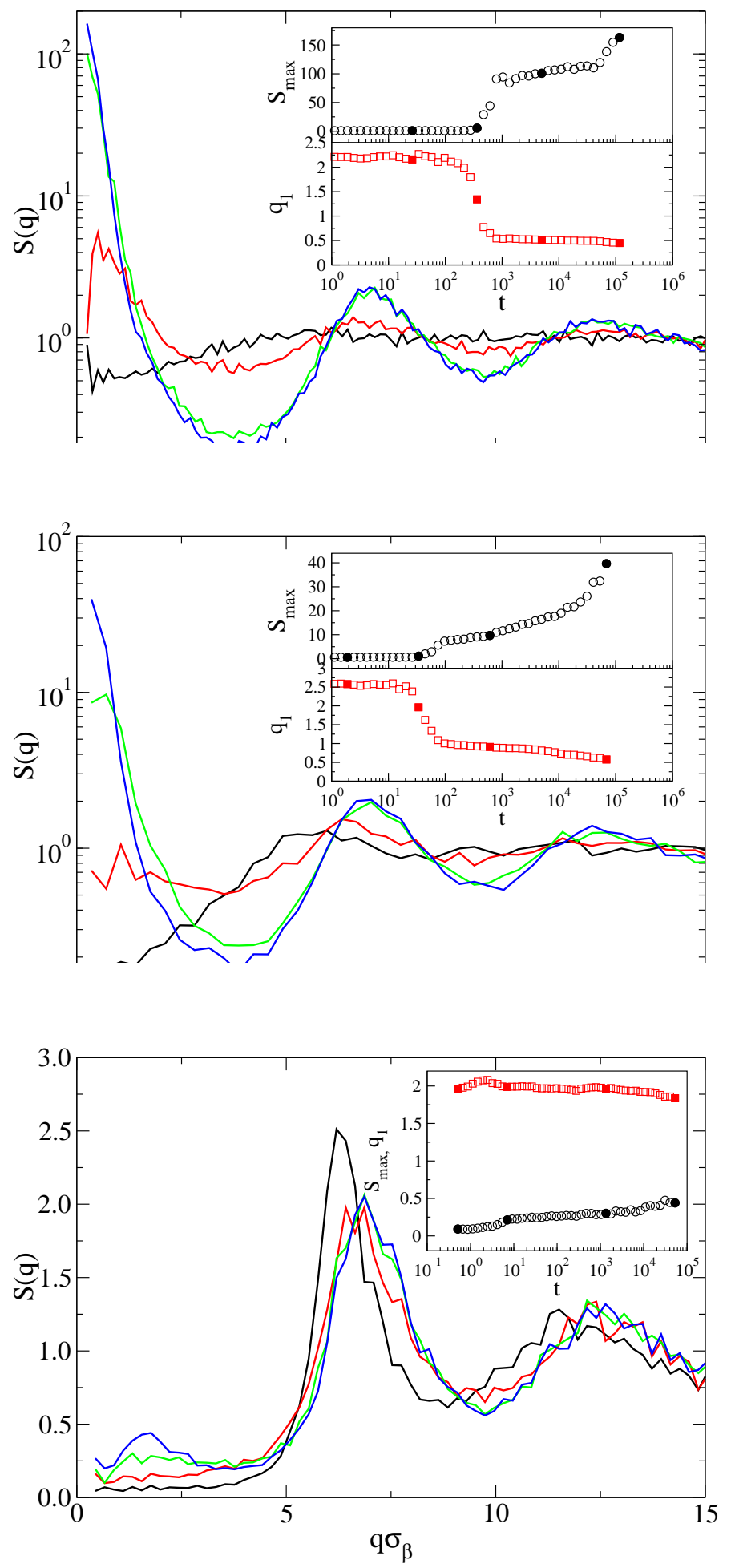

FIG. 8: Same as Fig 6 for $T_{f}=0.15$. 

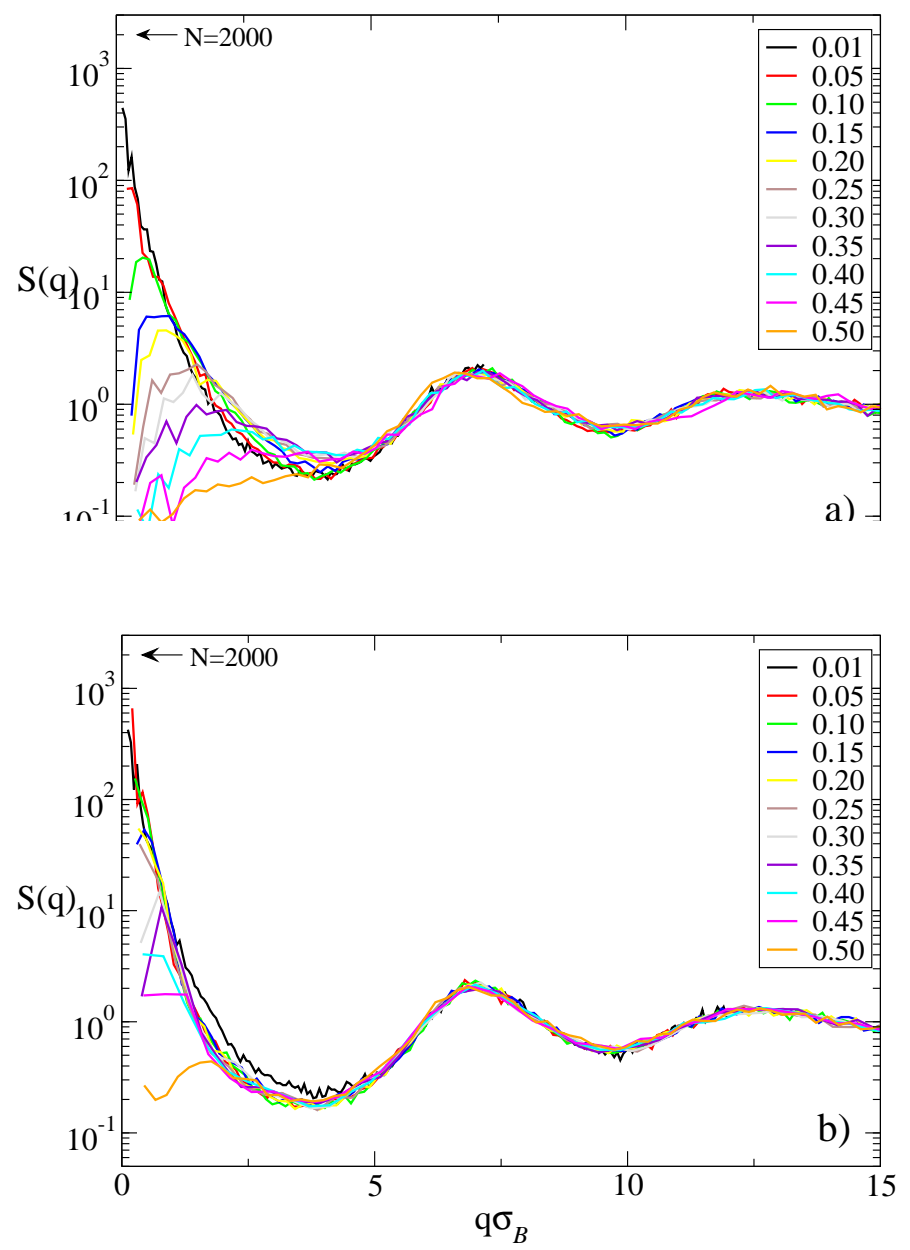

FIG. 9: Static structure factors $S(q)$ of the final configuration for different packing fractions. (a) $T_{f}=0.05$, (b) $T_{f}=0.15$

FIG. 10: Representation of the studied two dimensional slabs. The three slabs are chosen at an height $z=2.4, z=12.0$ and 22.6 and they have a width of $4 \sigma_{b}$

FIG. 11: Snapshots of the two dimensional slabs (see Fig [10) of the final configurations at three values of the $z$-coordinate. (a) $T_{f}=0.05$ and $\phi=0.10$; (b) $T_{f}=0.15$ and $\phi=0.10$ 

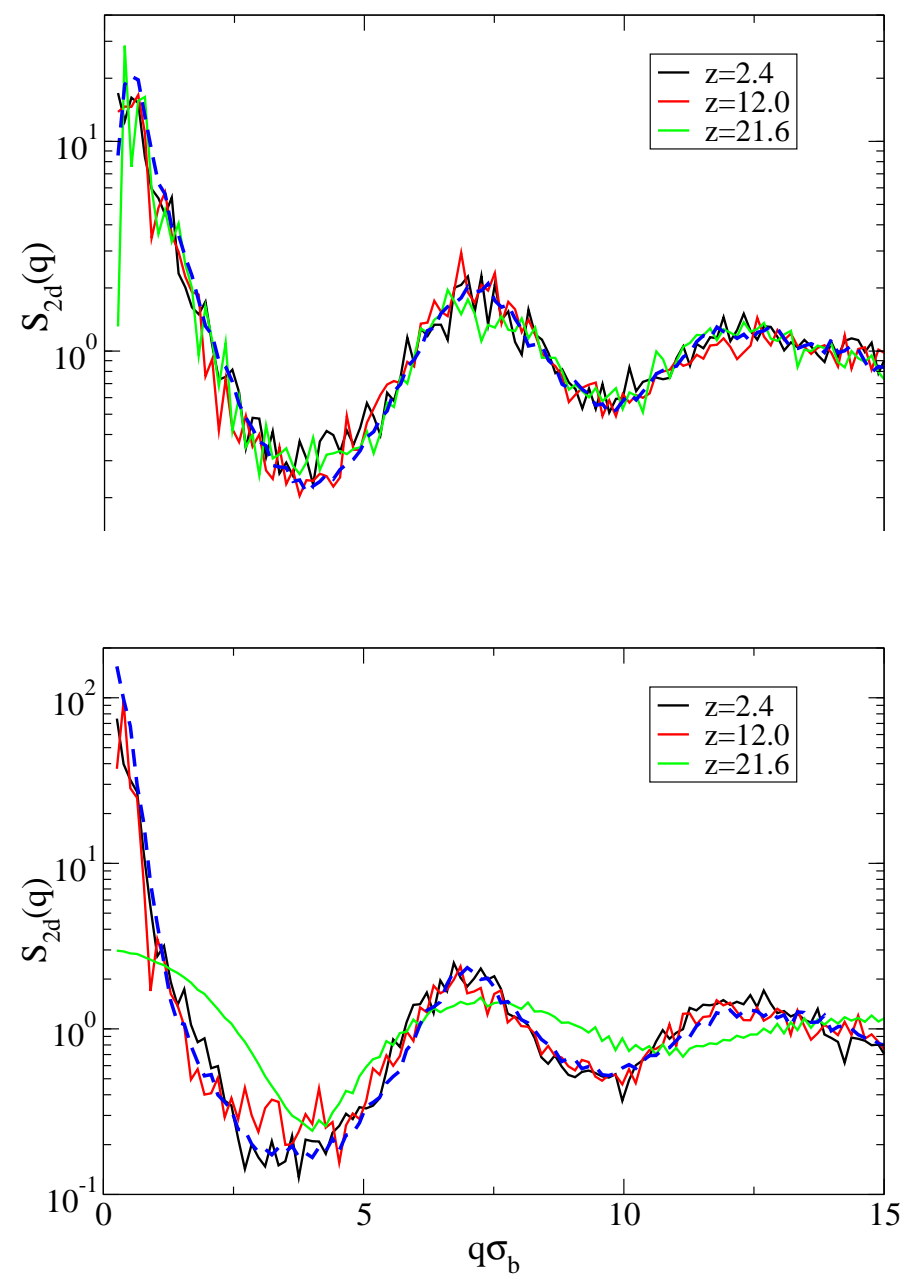

FIG. 12: Two dimensional structure factor $S_{2 d}(q)$ for different values of the $z$-coordinate (see text for details). (a) $T_{f}=0.05$ and $\phi=0.10$; (b) $T_{f}=0.15$ and $\phi=0.10$. The dashed line corresponds to the three dimensional spherically averaged $S(q)$. 

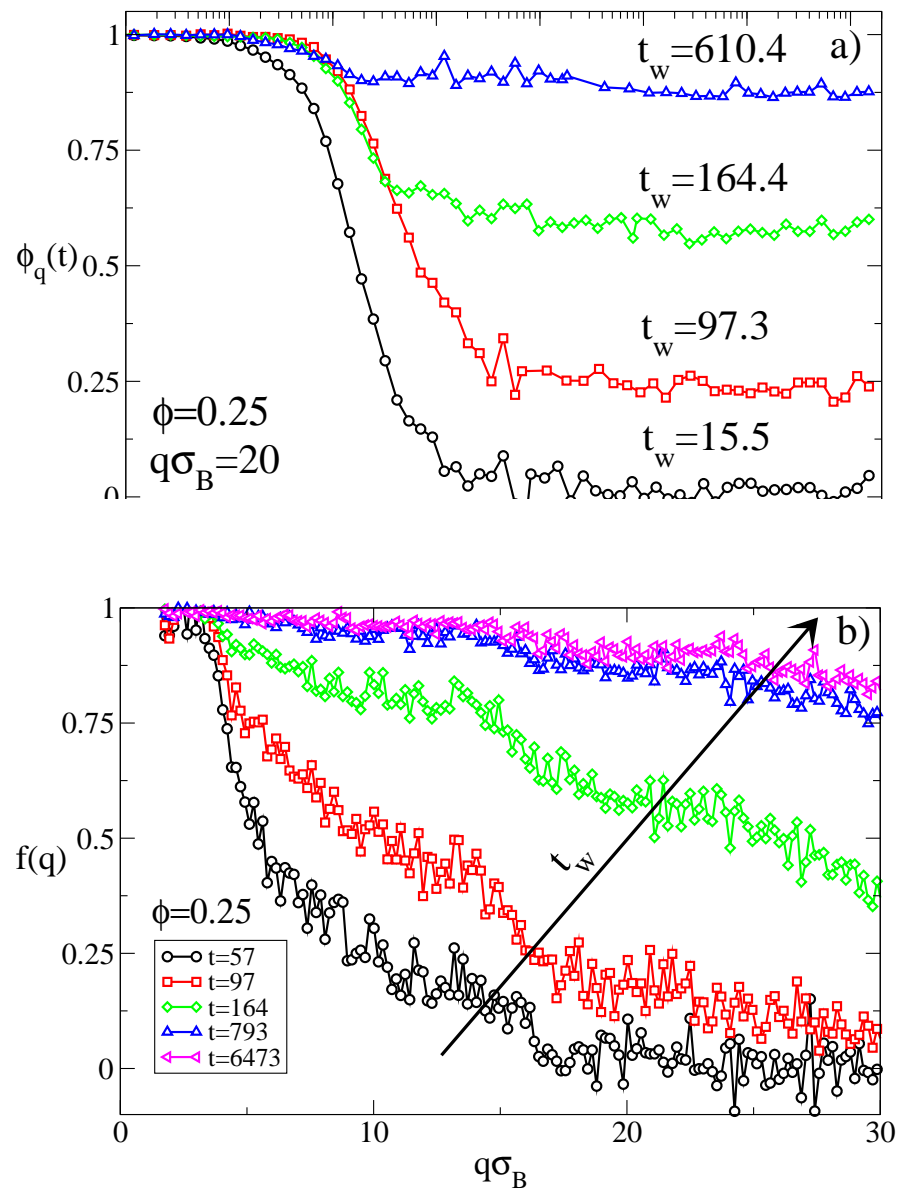

FIG. 13: a) Density-density correlation function $\phi_{q}(t)$ for different waiting times $t_{w}$ after the quench. This case corresponds to $\phi=0.25$ and $T_{f}=0.05$. Correlation functions are calculated for $q \sigma_{B}=20$. (b) Time evolution of the non ergodicity parameter $f_{q}$ with increasing $t_{w}$ for the same case. 

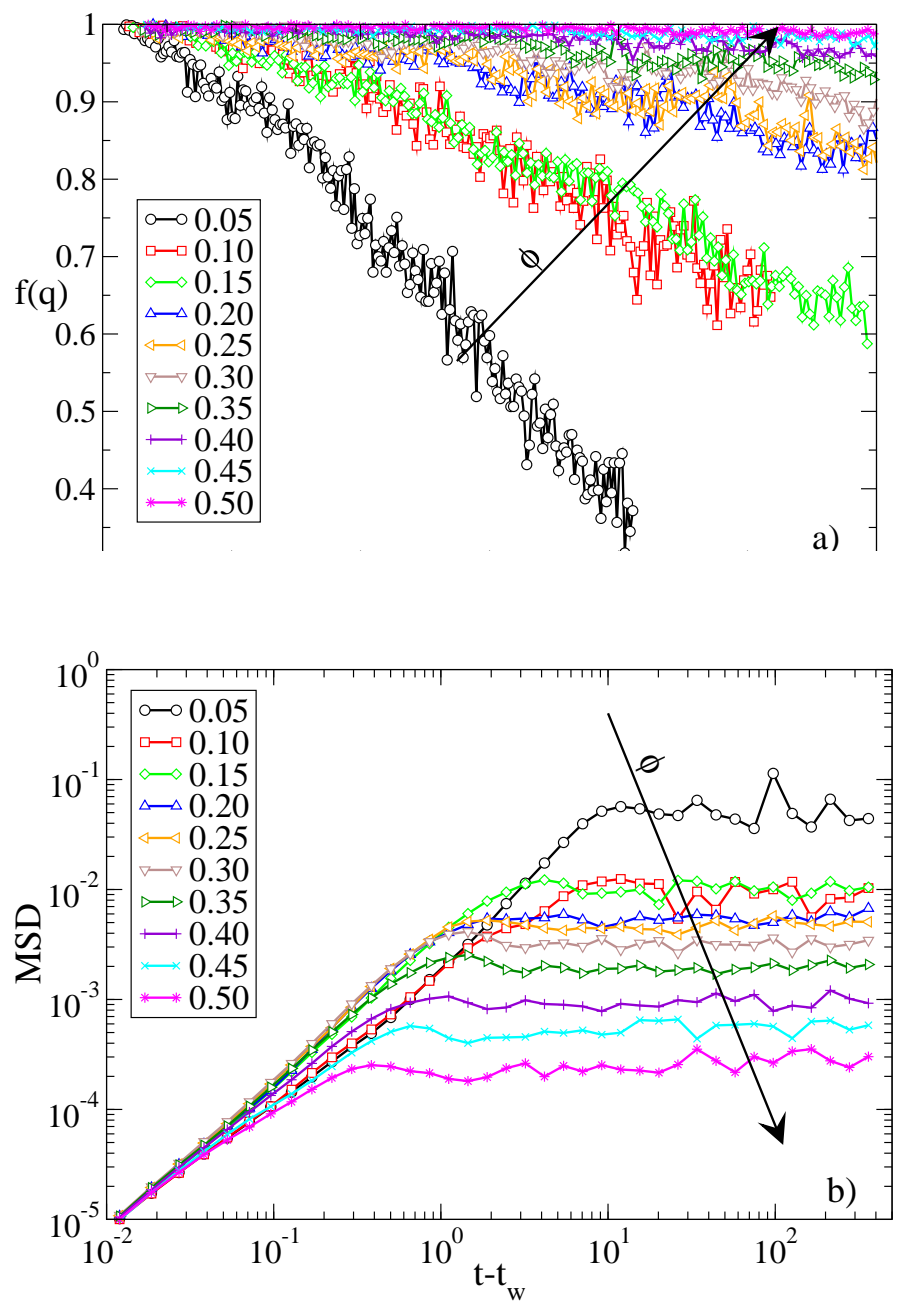

FIG. 14: a) Non ergodicity parameter for different packing fraction in the final arrested state, at $T_{f}=0.05$.

b) Mean square displacement in the final arrested state for different values of $\phi$. 